\title{
Gestión de una Cultura de Innovación Basada en las Personas
}

\author{
Jaime E. Souto ${ }^{1 *}$
}

Resumen: La cultura de innovación tiene un papel central en la capacidad de innovación de las organizaciones. Así como un destacable efecto sobre la competitividad. Ante su relevancia es indispensable determinar una serie de herramientas para adaptarla a las necesidades e idiosincrasia de cada empresa a lo largo de diferentes periodos de tiempo. En este trabajo se aborda la gestión de la cultura de innovación desde la dirección del personal y de los equipos de trabajo, fruto de reconocer el papel central de las personas en la cultura y la innovación. Inevitablemente, las personas deben ser el punto central en cualquier cultura corporativa, especialmente en su enfoque hacia la innovación. De hecho, una instrumentación efectiva en la creación y mantenimiento de un lugar de encuentro y expresión de las personas es un requerimiento ineludible.

Palabras clave: cultura de innovación; dirección del personal; trabajo en equipo; innovación.

\begin{abstract}
The innovation culture has a central role in the innovation capabilities of organizations, as well as a remarkable effect on competitiveness. Given its relevance, it is essential establishing a set of tools to suit the needs and idiosyncrasies of each company over different periods of time. In this paper, the management of innovation culture is approached from the direction of staff and work teams, as result of recognizing the central role of people in culture and innovation. Inevitably, people should be the central point in any corporate culture, especially in its approach toward innovation. In fact, an effective implementation in creating and maintaining a meeting point and expression of people is an indispensable requirement.
\end{abstract}

Keywords: innovation culture; staff management; teamwork; innovation.

Submitted June 5th 2015 / Approved October 7th 2015

\section{Introducción}

Actualmente, la innovación es fundamental para la competitividad y supervivencia de las empresas. Las empresas innovadoras sobrevivirán y prosperarán en detrimento de las demás (Schumpeter, 1934 y 1942).

Sin embargo, tanto la gestión de la innovación como la tarea de agrupar los esfuerzos de los miembros de la organización hacia ese fin no es sencillo. Las principales razones son debidas a su complejidad, riesgo e incertidumbre.

La cultura desempeña una labor trascendental en el proceso de innovación de las organizaciones, con un importante efecto sobre sus resultados (Feldman, 1988; Kiurunen, 2009; Lin, 2009; Martins y Terblanche, 2003; McLaughlin et al., 2008; Morcillo, 1997; Morcillo et al., 2007; Lee et al., 2008; Villena-Manzanares y Souto-Pérez, 2015).

La cultura corporativa tiene una gran influencia sobre la competitividad. Las organizaciones atesoran ventajas competitivas sostenibles resultado o consecuencia de su cultura corporativa. Mintzberg et al. (1998) destacan el papel de la cultura corporativa sobre la competitividad de las empresas y el mantenimiento de sus ventajas competitivas, al reconocerla como un recurso original, imposible de imitar y no sustitutivo. Cada entidad tiene una cultura única y, por tanto, una cultura de innovación diferente a la de las demás, con sus propias características y niveles de adaptabilidad, flexibilidad y convivencia con la incertidumbre y ambigüedad.

En torno a las personas como el determinante de la cultura de innovación en las organizaciones, este trabajo plantea y desarrolla dos pilares fundamentales de su creación y gestión.

Desde una perspectiva centrada en los miembros de la organización y en el imprescindible papel de las personas en la innovación y creatividad, sus esfuerzos y compromiso con la innovación son cruciales en la construcción de organizaciones capaces de conseguir que la innovación y la creatividad fluyan de forma natural.

Las condiciones necesarias para posibilitar un entorno propicio para la innovación y la creatividad en las organizaciones requiere del entendimiento de las organizaciones como lugares de encuentro y colaboración entre personas, favorables para aunar esfuerzos e intercambiar experiencias. Es imposible plantear los dos pilares comentados, sin plasmar al mismo tiempo en ellos las bases necesarias para favorecer el encuentro y colaboración entre las personas.

1 Universidad Autónoma de Madrid. Departamento de Dirección de Empresas.

*Email: jaime.souto@uam.es. 


\section{Proposición de un Modelo de Gestión de la Cultura de In- novación}

La cultura es un conjunto de asunciones básicas compartidas entre las personas, capaces de determinar sus comportamientos y relaciones (Schein, 1992). Schein (1992) estudia la cultura como una consecuencia del aprendizaje de un grupo, al solucionar los problemas externos de adaptación y los internos de integración. En la cultura de innovación esas soluciones son fruto del descubrimiento y la creatividad, resultado de enfrentarse al riesgo e incertidumbre de romper con lo establecido y cotidiano. Bajo esa perspectiva el elemento central de la cultura de innovación son las personas y su trabajo en grupo, constantemente orientado hacia la consecución de innovaciones.

Por tanto, es posible identificar dos aspectos básicos en la gestión de una cultura de innovación: la dirección de las personas y el trabajo en equipo.

\section{Dirección del Personal}

Las empresas no innovan por accidente, sino gracias a una adecuada orientación de todos sus empleados hacia ese fin. Además, ante la creciente importancia de los intangibles en la mejora de la situación competitiva de las organizaciones, en las personas yace el éxito de muchas compañías, especialmente, al ser depositarias de muchos conocimientos fruto de sus experiencias y vivencias. Así, la dirección del personal tiene una relevancia critica en el desarrollo de una cultura de innovación, al permitir la dirección, motivación y recompensa de su gente en la consecución de innovaciones.

El clima de creatividad y comportamiento innovador deseado de los empleados es factible con una adecuada implementación de los sistemas de dirección del personal. Los sistemas de dirección del personal incluyen: selección y reclutamiento, formación, recompensa (Kono, 1990) y valoración y gestión del desempeño.

\section{Sistema de Selección y Reclutamiento}

Tan importante como reclutar a las personas con las habilidades, conocimientos y experiencia necesarias para el puesto a desempeñar, es la capacitación de esos individuos para innovar. Pues la innovación es una actividad que involucra a toda la organización, cada trabajador debe aportar su grano de arena.

La identificación, selección y reclutamiento de los individuos apropiados, junto con una amplia variedad de habilidades profesionales y áreas de conocimiento, son el caldo de cultivo de la innovación.

Pero los nuevos miembros de la organización no sólo se deben seleccionar y reclutar por su perfil individual, sino también por su aportación a la diversidad de la entidad, al permitir la entrada de nuevos conocimientos que se puedan combinar con los existentes, en otras palabras, nuevos empleados realmente capaces de trabajar con los actuales y de aportar nuevas perspectivas. Así, un nuevo individuo no sólo debe ser valorado por sus habilidades y cualificaciones para su puesto, sino también por su aportación a toda la compañía y a los grupos de trabajo en donde se integrará.
Además, los rasgos de personalidad del individuo y como encajan con sus compañeros son una variable a tener mucho más que presente, de gran relevancia, al incluir cuestiones tan importantes para la cultura de innovación como la tolerancia al riesgo, independencia y creatividad.

Con todos esos aspectos es posible seleccionar y reclutar el tipo adecuado de empleado, posibilitando el trabajo en equipo y la creación de equipos equilibrados en la mezcla de habilidades. Incluso posibilita el realineamiento de un equipo creativo, al introducir nuevos miembros (Bilton, 2007). Para crear y mantener una cultura innovadora, es necesario reclutar el tipo correcto de empleado (Kirkbride, 1987), sin ese elemento básico es imposible dar forma a una cultura innovadora. A menudo, el fracaso de una compañía o un proyecto se puede explicar por reclutar a la clase equivocada de empleados (Martin, 1984).

Pero el primer paso antes de seleccionar y reclutar nuevos trabajadores es una buena determinación de las necesidades de personal para innovar, tanto de su cantidad como de sus características en términos de habilidades, conocimientos, experiencia y área de conocimiento.

\section{Formación}

El desempeño de un trabajador no depende únicamente de reclutar a la persona más apropiada para un puesto, ni tampoco de una adecuada gestión de sus habilidades y destrezas, también es imprescindible dotarlos de nuevas habilidades y conocimientos, posibilitando el crecimiento personal del individuo hacia lo que quiere ser y hacer, reduciendo el desfase entre lo que puede y sabe hacer y lo que quiere poder y saber hacer.

Esa es la función de la formación, mejorando los conocimientos y destrezas de los trabajadores, tanto al mantenerlos actualizados como al incrementarlos. Así, es posible aumentos considerables en el desempeño de un puesto de trabajo, posibilitando la innovación.

Pero la principal contribución de la formación a la cultura de innovación no se restringe a dotar de los conocimientos necesarios para innovar, hay otro punto critico para la cultura de innovación, consistente en contar con personas de mente abierta, capaces de ver más allá de lo evidente. Muchas innovaciones son fruto de conocimientos ya existentes que son combinados o empleados de forma original. Aunque ninguna persona es innovadora si no cuenta con las habilidades y conocimientos necesarios para serlo, por supuesto, tras su capacitación, es imprescindible la motivación hacia este comportamiento. Por tanto, ambas se complementan y son imprescindibles para la innovación y el establecimiento de una cultura de innovación.

Pero tan importante como formar es hacerlo adecuadamente, es decir, en temas pertinentes. Los nuevos conocimientos y habilidades deben ser relevantes para las tareas y funciones desarrolladas en el puesto actual o en otros futuros. Además, esta segunda posibilidad abre la puerta al incremento de la flexibilidad de asignación de los empleados, algo no sólo de gran valor para la adaptación de la 
compañía a los cambios en el entorno, sino también al intercambio de conocimientos en la entidad, especialmente los tácitos, difícilmente replicables.

Contar con empleados polivalentes, capaces de desempeñar otras funciones y tareas, es un aspecto elemental que posibilita la reestructuración de la empresa y su adaptación a los cambios. En un entorno económico con una considerable aceleración de la aparición de cambios como el actual, la capacidad de adaptación es una variable critica para la competitividad.

Además, la formación del personal es uno de los factores involucrados en la creación de una cultura innovadora (Attwood, 1990).

\section{Sistema de Valoración y Gestión del Desempeño}

El cometido de este sistema no se restringe a la evaluación del trabajo de los empleados y su aportación a la consecución de innovaciones, también incluye la gestión de sus aptitudes y actitudes. Este planteamiento enfatiza la importancia de la gestión de las competencias atesoradas por las personas.

Las personas son factores clave para el éxito y la consecución de los objetivos de las organizaciones, al brindar sus conocimientos y habilidades. Ninguna entidad debe desaprovechar el talento de sus empleados, ni dejar de explotar sus conocimientos y habilidades.

Tanto las actitudes como las aptitudes de las personas que conforman una organización tienen un gran valor. La actitud de cada individuo está determinada por lo que quiere ser y hacer, de forma colectiva, su agregación e interacción son críticas para la configuración de una cultura de innovación, al marcar las voluntades y motivación del grupo. Por su parte, la aptitud de cada individuo marca que puede y sabe hacer fruto de sus conocimientos tácitos y explícitos que afloran en forma de habilidades y destrezas.

Tanto las aptitudes como las actitudes conforman roles de comportamiento e interacción entre los individuos, así, son la piedra angular de la cultura de innovación. Además, es indudable la riqueza cultural fruto de aunar actitudes y aptitudes, no todos pensamos ni vemos el mundo igual, tampoco tenemos los mismos conocimientos y habilidades. Sin duda, una de las claves del éxito y aparición de muchas innovaciones ha sido la combinación de diferentes perspectivas y puntos de vista sobre el entorno de actividad y el propio negocio, como por ejemplo sobre el entendimiento de un negocio o una actividad.

Por tanto, la diversidad en una compañía no es fruto de juntar cosas diferentes por azar o bajo su aparición por casualidad, sino de aunar e integrar laboriosamente cosas diferentes, siempre bajo una constante gestión de todas las competencias de los miembros de la organización.

Aunque las actitudes son menos evidentes y difíciles de identificar, su valor en la creación de una cultura de innovación fuerte y efectiva son evidentes. Un comportamiento y orientación hacia la innovación sólo es posible con una alineación de las voluntades y motivación de los individuos hacia esta actividad. En concreto, es crucial la motivación para enfrentarse a los diferentes problemas del proceso de innovación, como son el riesgo y la incertidumbre.

\section{Sistema de Recompensa}

Por su impacto sobre el comportamiento de los individuos y grupos, es de gran utilidad en la gestión de una cultura de innovación, tanto en las fases de creación y consolidación como en la de mantenimiento. La principal razón del impacto de este sistema se debe a la tendencia hacia la desaparición de los comportamientos no recompensados o castigados (Van de Ven et al., 1989).

Para convertir este sistema en una valiosa herramienta en la gestión de una cultura de innovación, la consecución de innovaciones y comportamientos encaminados hacia ese fin deben ser recompensados. Así, tanto las actividades innovadoras como la demostración de compromiso con los valores concordantes con la cultura de innovación serán recompensados (Milton et al., 1992).

Además, las recompensas han de compensar los riesgos asumidos por los innovadores. Si no fuera así, el nivel de riesgo asumido por estos individuos seria bajo, provocando un nivel bajo de innovación (Amabile, 1988). Por tanto, el sistema de recompensas no castigará los fallos inevitables y parte del proceso de aprendizaje, aunque impliquen un coste en dinero y tiempo para la organización. Penalizar los errores cometidos desemboca en la eliminación de la iniciativa (Coyne, 1990).

Las recompensas se pueden clasificar principalmente en los siguientes tres tipos: financieras, promoción y reconocimiento.

Las financieras consisten en cuantías económicas. Aunque son poderosas motivadoras, no siempre son las más efectivas, especialmente, cuando el nivel retributivo actual cubre sobradamente cualquier necesidad económica.

La promoción en un ascenso del trabajador. Sin embargo, no puede ser entregada a todo el mundo, ni de forma igualitaria, sólo debe ser utilizada excepcionalmente.

El reconocimiento del esfuerzo innovador es una de las recompensas más significativas, por su importante contribución a la gestión y fomento de una cultura innovadora. El reconocimiento profesional y trabajar en áreas con grandes desafíos puede ser un potente motivador (Lank, 1997). El reconocimiento tiene algunas ventajas con respecto a otras recompensas, se puede dar en términos generales y más frecuentemente.

Una adecuada combinación de estos tres tipos de recompensas posibilita la motivación de los trabajadores. Aunque es muy importante enfocarlo tanto a nivel individual como grupal, para estimular los mejores resultados de cada persona, como por ejemplo la colaboración e integración dentro de los grupos de trabajo en que este ubicado.

Una incorrecta implantación del sistema de recompensas puede tener un efecto contrario al deseado. Un reconocimiento desigual 
o inadecuado puede ser contraproducente en el fomento de la innovación (Rydz, 1986).

\section{Sistema de Plan de Carrera}

Este sistema está muy relacionado con el de formación, al aunar las ambiciones profesionales de los individuos con las metas de la organización, proporcionando la educación y formación necesaria para permitir el progreso de la carrera profesional dentro de la organización.

La aportación a la cultura de innovación consiste en posibilitar el desarrollo profesional a partir del desempeño innovador de los individuos, nuevamente vuelve a estar relacionado con otro sistema, el de valoración del desempeño.

Pero no sólo se relaciona con unos cuantos de los restantes sistemas de dirección del personal, también enfatiza la relevancia de integrar de forma coherente toda la dirección del personal, pues los diferentes sistemas no funcionaran de forma aislada de los demás. La dirección del personal orientada hacia la cultura de innovación es como una mesa con cuatro patas, si cualquiera de las patas es más larga o corta a las demás, la mesa se tambalea y resulta inservible, si una es más débil a las demás, la carga soportada por todas terminará por los suelos.

Por tanto, los cuatro sistemas constitutivos de la dirección del personal deben estar equilibrados y coordinados, sino lo conseguido por uno será perdido por otro.

\section{Gestión del Trabajo en Equipo}

El trabajo en equipo ha tomado gran relevancia en las compañías, por su papel en la consecución de innovaciones, al facilitar o posibilitar la creación, transferencia, asimilación y combinación de conocimiento, tanto explícitos como tácitos.

En el seno de los grupos son generados recursos culturales, capaces de apuntalar las ventajas competitivas de la empresa (Wernerfelt, 1989).

No es suficiente con hacer trabajar juntas a las personas, tienen que cooperar e interactuar en un ambiente abierto y participativo. Un equipo únicamente puede tener éxito si sus miembros aúnan esfuerzos, conocimientos y habilidades hacia los objetivos comunes. Sin duda, el primer paso es alinear los objetivos de todos los miembros del equipo, a través de la selección de aptitudes y actitudes afines y complementarias es posible. Aunque las diversas desviaciones que se irán produciendo a lo largo del tiempo se deberán corregir, bien con la modificación de los miembros del equipo, dando entrada a nuevos individuos o recolocando a otros, o bien con la realineación de los objetivos de algunos miembros o la modificación de los objetivos de todo el grupo.

La dirección dirige las relaciones dentro de los grupos innovadores, re-ensamblando a sus miembros dentro de nuevas configuraciones que posibiliten el proceso de innovación. Cada miembro de un grupo juega un papel peculiar en el mismo, tanto en su adecuación a la labor realizada como su aportación a la orientación de ese equipo de trabajo, determinando su composición y relaciones su propensión hacia el impulso de la innovación en la conquista de las metas de la organización.

Además, de la mezcla de especializaciones y áreas resulta la fertilización cruzada de ideas, una característica fundamental de las organizaciones innovadoras (Gerstenfeld, 1970; Kanter, 1988). Esa interdisciplinariedad es posible gracias al trabajo en equipo de personas procedentes de diversas disciplinas, incentivadas para colaborar.

Una cultura de innovación fuerte requiere de procesos de innovación estructurados en torno a los grupos basados en la interdisciplinariedad. Sólo así, será posible la consecución de innovaciones generadas a partir de conocimientos procedentes de diferentes disciplinas, posibilitando la puesta en común y combinación de los mismos, así como, futuros desarrollos de los mismos.

En la actualidad la innovación es una tarea de todos los miembros de la organización, ya no puede estar únicamente encerrada en un departamento, zona, área funcional o unidad de negocio de una empresa. La cooperación inter-funcional es la más fructífera para la innovación (Nonaka y Takeuchi, 1995). En definitiva, todos deben ser participes, todos deben aportar su grano de arena para lograr innovaciones.

Este aspecto de agrupar a todas las personas hacia la obtención de innovaciones es el mantra de la cultura de innovación. Todos deben hacer su aportación y poner sus conocimientos y habilidades al servicio de conseguir innovaciones. En el contexto actual, ante un creciente y muy relevante efecto de la innovación sobre la competitividad de las empresas, innovar es una prioridad si la compañía quiere tener éxito y sobrevivir. Las empresas no innovadoras languidecerán y finalmente desaparecerán (Schumpeter, 1934).

\section{Conclusiones}

La cultura de una corporación depende de aspectos sociológicos e ideológicos, así como de los medios disponibles. La primera hace referencia a la comunicación entre individuos. La segunda a las creencias, rituales, prácticas y mitos. Por último, los medios están relacionados con los procesos, técnicas y herramientas disponibles, determinados por el avance científico y tecnológico.

El modelo planteado posibilita la gestión de la cultura de innovación a través de los aspectos sociológicos e ideológicos de la cultura corporativa. Así, de forma acompasada los pilares expuestos actúan sobre:

(1) Las relaciones y comunicación entre las personas.

(2) Las creencias, rituales y prácticas admitidas por el colectivo de la compañía.

Una organización proclive hacia la innovación, capaz de generar nuevos descubrimientos y comercializarlos con éxito, no surge por 
casualidad, es fruto del tesón de sus miembros, quiénes han de ser orientados convenientemente con respecto a la situación actual. De esa forma, es posible mantener un espíritu innovador en situaciones complicadas para la empresa, aprovechando las oportunidades existentes y haciendo frente a las amenazas reales.

No obstante, en algunas compañías van más allá, fundamentando su gran éxito en convertir amenazas de su entorno en oportunidades. Algo impensable para muchas entidades, pero viable al gestionar convenientemente la cultura de innovación desde los pilares detallados.

La dirección del personal y el trabajo en equipo permiten la gestión de una cultura de innovación, e incluso, su creación, a través de las personas que se ven involucradas, pero su gestión y dirección por sí sola, sin tener en cuenta a las personas, no permite crear y mantener la cultura deseada. Por tanto, estas herramientas no son elementos constitutivos de una cultura de innovación, sólo los aspectos sociológicos, ideológicos y los medios disponibles por el colectivo de la entidad lo son. Sin embargo, sí actúan sobre esos aspectos, constituyendo una vía para dar lugar a comportamientos basados en asunciones básicas proclives hacia la innovación.

El valor de cualquier herramienta de gestión de una cultura de innovación debe medirse por su efecto sobre el comportamiento de las personas. La propuesta presentada, trabaja sobre los individuos y grupos, con el objetivo de suscitar pautas de conducta propensas a la innovación.

Las herramientas de dirección del personal son elementales en la creación de empresas más competitivas, al ser el pilar básico en la creación y mantenimiento de una cultura de innovación. Pero también permite mejorar la flexibilidad de toda la compañía.

Una de las principales barreras a la innovación y al establecimiento de una cultura de innovación es la inercia y la resistencia al cambio. Para conseguir que los empleados asuman la innovación y el cambio como algo natural, es esencial hacerlos participes, aquí la formación y gestión por competencias toman un papel crucial.

Una cultura de innovación no se crea a partir de unidades separadas para explotar el presente y desarrollar el futuro, tal como predica la innovación ambidiestra, ambas deben ir de la mano y es imprescindible su colaboración para evitar errores y fracasos innecesarios. Pero la eliminación de la resistencia al cambio no es posible sin hacer participes del nuevo proyecto a los miembros de los actuales, especialmente, si ese nuevo proyecto del cual están desvinculados y no pueden aportar nada termina por sustituir a los vigentes.

Los cambios reconocidos como oportunidades por la dirección de una compañía deben serlo también para el resto de personas de la compañía. Una oportunidad para la empresa se convierte en una amenaza para sus empleados si se ven relegados y desplazados, algo evitable si se utilizan adecuadamente los sistemas de dirección del personal. Una adecuada gestión de las competencias de las personas y su formación serán vitales para recolocar a los empleados afectados por el nuevo proyecto, pero sobre todo, para aprovechar sus conocimientos y habilidades en la buena consecución y éxito de ese proyecto, obteniendo el mejor resultado posible.

Sin embargo, esto no implica que la existencia de unidades separadas no sea útil, pero su uso debería limitarse al desarrollo de diferentes caminos dependientes -algo olvidado en la actual literatura. Aunque siempre aprovechando sinergias entre ambas unidades, en buena parte posibles, si la compañía dispone de personas polivalentes y con capacidad de aprender.

Sin duda la gestión de la cultura de innovación hace hincapié en la importancia de las personas proclives al aprendizaje, con capacidades para aprender constantemente y posibilitar el surgimiento de nuevos conocimientos o la combinación de los existentes en nuevas direcciones.

Enfocándose sobre el factor humano es posible crear una espiral virtuosa en la mejora del desempeño de la organización, llegando incluso, a poder crear una verdadera organización capaz de aprender. Una organización de esas características, tiene a las personas presentes en todo lo que hace, pues ellas son el corazón del aprendizaje de la organización. La cultura de innovación es el elemento central en la creación de una organización capaz de aprender, en donde las personas son lo más apreciado. La cultura de innovación incluye a la "cultura de aprendizaje", orientándola tenazmente hacia la obtención de innovaciones dentro de la dirección de progreso seleccionada por la entidad.

Pero la innovación no es una actividad individual, requiere del trabajo en equipo. Sólo aglutinando los esfuerzos de toda la compañía se puede tener éxito en esa actividad.

La gestión del trabajo en equipo es el factor clave para conseguir un lugar de encuentro entre las personas. Una forma de hacer trabajar juntos a los individuos hacia un fin colectivo, mientras comparten y ponen en común sus conocimientos, posibilitando su aplicación, generación y diseminación por toda la empresa.

Por tanto, la gestión de una cultura de innovación a lo largo del tiempo implica fundamentalmente una adecuada dirección del personal y del trabajo en equipo. Aunque ambos pilares deben enfocarse siempre hacia la creación de un lugar de encuentro y expresión de las personas, para posibilitar la generación e impulso de ideas y cambios encaminados a la mejora de la eficiencia y eficacia.

\section{Referencias}

Amabile, T.M. (1988). A model of creativity and innovation in organizations. Research in Organizational Behavior, 10, 123-167.

Attwood, T. (1990). Corporate culture: For and against you. Management Accounting, 68(1), 26-29. 
Bilton, C. (2007). Management and creativity. Oxford, UK: Blackwell Publishing.

Coyne, W. (1990). Innovation as a competitive advantage. Hospitals, 64(10), 88-88.

Feldman, S.P. (1988). How organizational culture can affect innovation. Organizational Dynamics, 17(1), 57-68. http://dx.doi. org/10.1016/0090-2616(88)90030-7

Gerstenfeld, A. (1970). Effective management of research \& development. Massachusetts: Addison-Wesley.

Kanter, R.M. (1988). When a thousand flowers bloom: Structural, collective, and social conditions for innovation in organizations. En B.M. Staw y L.L. Cummings (Eds.), Research in organizational behavior (pp. 169-211). Greenwich, CT: JAI Press.

Kirkbride, P.S. (1987). Personnel management and organisational culture: A case of deviant innovation?. Personnel Review, 16(1), 3-9. http://dx.doi.org/10.1108/eb055552

Kiurunen, A.M. (2009). Culture effect on innovation level in European countries. International Journal of Business Innovation and Research, 3(3), 311-324. http://dx.doi.org/10.1504/ijbir.2009.024182

Kono, T. (1990). Corporate culture and long-range planning. Long Range Planning, 23(4), 9-19. http://dx.doi.org/10.1016/00246301(90)90148-w

Lank, E. (1997). Leveraging invisible assets: The human factor. Long Range Planning, 30(3), 406-412. http://dx.doi.org/10.1016/s00246301(97)90258-2

Lee, C.K., Tan, B., y Chiu, J.Z. (2008). The impact of organisational culture and learning on innovation performance. International Journal of Innovation and Learning, 5(4), 413-428. http://dx.doi. org/10.1504/ijil.2008.017561

Lin, L.H. (2009). Effects of national culture on process management and technological innovation. Total Quality Management \& Business Excellence, 20(12), 1287-1301. http://dx.doi. org/10.1080/14783360903250621

Martin, M.J.C. (1984). Managing technological innovation and entrepreneurship. Reston, VA: Reston Publishing Company.

Martins, E.C., y Terblanche, F. (2003). Building organisational culture that stimulates creativity and innovation. European Journal of Innovation Management, 6(1), 64-74. http://dx.doi. org/10.1108/14601060310456337
Mclaughlin, P., Bessant, J., y Smart, P. (2008). Developing an organisation culture to facilitate radical innovation. International Journal of Technology Management, 44(3-4), 298-323. http://dx.doi. org/10.1504/ijtm.2008.021041

Milton, D., Verran, J., Murdaugh, C., y Gerber R. (1992). Differentiated group professional practice in nursing: A demonstration model. The Nursing Clinics of North America, 27(1), 23-30.

Mintzberg, H., Ahlstrand, B., y Lampel, J. (1998). Strategy Safari. New York: Free Press.

Morcillo, P. (1997). Dirección estratégica de la tecnología e innovación. Madrid: Civitas.

Morcillo, P., Rodriguez, J. M., y Rubio, L. (2007). Corporate culture and innovation: In search of the perfect relationship. International Journal of Innovation and Learning, 4(6), 547-570. http://dx.doi. org/10.1504/ijil.2007.013448

Nonaka, I., y Takeuchi, H. (1995). The knowledge-creating company: How Japanese companies create the dynamics of innovation. New York: Oxford University Press.

Rydz, J.S. (1986). Managing innovation. Massachusetts: Ballinger Publishing.

Schein, E.H. (1992). Organizational culture and leadership. San Francisco: Jossey-Bass.

Schumpeter, J. (1934). Theory of economic development: An inquiry into profit, capital, credit interest, and business cycle. Cambridge: Harvard University Press.

Schumpeter, J. (1942). Capitalism, socialism and democracy. London: Unwin University Books.

Van de Ven, A.H., Angle, H.L., y Poole, M. S. (1989). Research on the management of innovation. New York: Harper \& Row, Ballinger Division.

Villena-Manzanares, F., y Souto-Pérez, J.E. (2015). The impact of strategic intangible on the export performance of the manufacturing SMEs. Intangible Capital, 11(1), 13-40. http://dx.doi.org/10.3926/ic.567

Wernerfelt, B. (1989). From critical resource to corporate strategy. Journal of General Management, 14(3), 4-12. 